\title{
Many-particle density-matrix approach to a quantum dot system for the strong electron accumulation case
}

\author{
K. M. Indlekofer and H. Lüth \\ Institut für Schicht- und Ionentechnik, Forschungszentrum Jülich GmbH, 52425 Jülich, Germany
}

(Received 18 April 2000)

\begin{abstract}
We consider the system of an electronic quantum dot with a base set of discrete single-particle levels due to quantization effects in an arbitrarily given attractive potential. Intradot electron-electron interaction is described employing the full many-particle Coulomb interaction Hamiltonian in second quantization. Interaction effects arising from a capacitive response of the environment is incorporated within the framework of a classical interaction term. Hereby the environment consists of thermodynamical electron reservoirs coupled to the quantum dot system via weak tunnel barriers. Using this quantum dot model Hamiltonian we present a many-particle density-matrix approach in order to describe the thermodynamical state of the many-electron system and calculate expectation values of observables such as particle number and total spin. In the following we assume that exactly one reservoir dominates concerning a very weak particle injection. The other reservoirs are thought of as negligible tunneling probes. Especially the system of a laterally confined sub- $\mu \mathrm{m}$ resonant tunneling diode in the single-electron tunneling regime for the case of strong barrier asymmetry will be discussed as an example. Numerical results for realistic diode parameters suggest the definition of a capacitive and atomic regime of such an interacting quantum dot system.
\end{abstract}

\section{INTRODUCTION}

Continuously ongoing downscaling in conventional semiconductor microelectronics and the design of novel quantum effect based nanosized devices stimulate the theoretical research in the field of interacting few-electron systems and single-electron transport. Quantum dots represent an ideal model system to study quantum effects due to size quantization, tunneling, electron-electron interaction, and exchange and correlation in mesoscopic many-particle systems.

These quantum dots have been realized experimentally in a variety of semiconductor heterostructures and metallic systems. To name a few examples, mesoscopic systems within two-dimensional (2D) electron gases, laterally confined sub$\mu \mathrm{m}$ resonant tunneling diodes (RTD) or epitaxially grown islands have been investigated extensively. ${ }^{1}$

Various physical principles contribute to the electronic properties of such a quantum dot system, which are manifested in phenomena such as single-electron tunneling staircase characteristics and Coulomb blockade effects. ${ }^{2}$ First of all we have to account for discrete single-particle levels due to size quantization effects. Furthermore the electronelectron interaction together with the fermion nature of the electron (the Pauli principle roughly spoken) plays an important role.

Electronic transport through a quantum dot, which is coupled to electron reservoirs via tunnel barriers, implies a nonequilibrium state of the whole system, which is reflected in form of an electrical current between reservoirs of different chemical potential. ${ }^{3}$ A general approach consists of a many-particle nonequilibrium description by use of real-time Green's functions. ${ }^{4-6}$ Tunnel barriers often are described within the scope of tight binding models. ${ }^{7-9}$ Furthermore the intradot electron-electron interaction can be accounted for from a quantum field theoretical point of view to some de- gree. Thus mean-field approximations are commonly employed. For example, a Hartree self-consistent approach resembles a first-order mean-field approximation of electronelectron interaction terms. This semiclassical approach to interaction terms corresponds to a description within the scope of a capacity model, the so-called orthodox theory. ${ }^{10} \mathrm{~A}$ very successful equilibrium approach to the special case of a quantum dot system at $T=0$ represents the Hartree-Fock approximation where many-particle correlations are neglected. ${ }^{11,12}$

In this paper we focus on a description of the electronelectron interaction inside a quantum dot in the framework of many-particle (quantum field) statistics. $^{13,14}$ The general thermodynamical state is formulated by use of a projected many-particle density matrix in second quantization. Hereby we restrict ourselves to the case of a quantum dot, which is nearly in equilibrium with a weakly coupled electron reservoir. To a good approximation we can assume an equilibrium form of the density matrix with a given temperature and chemical potential. Additionally coupled reservoirs in an experimental setup must represent very weak tunnel probes for a characterization of electronic properties via electronic transport. Their influence on the quantum dot state therefore should be negligible with regard to a large class of observables such as particle number, spatial charge density distribution, and total spin. Obviously the current operator does not belong to this class. For $k_{B} T>\Gamma$ (where $-\Gamma / 2$ denotes the total imaginary part of the coupling self-energy induced by all reservoirs) we can neglect level broadening inside the quantum dot. The subsequent sections present a short description of our approach followed by some numerical results for the special case of a laterally confined sub- $\mu$ resonant tunneling diode in the strong accumulation singleelectron regime. 


\section{MANY-PARTICLE QUANTUM DOT MODEL}

The whole system under consideration consists of an interacting quantum dot and a number of electron reservoirs, which are coupled to the dot system via tunnel barriers. Each reservoir is assumed to be in a thermodynamical state with a given temperature and chemical potential. Obviously, the coupled system is in a nonequilibrium state for nonuniform temperature and chemical potential. This implies an electronic transport between the quantum dot and the adjacent reservoirs, which inject particles. In general, the state of such a system is given by a many-particle density matrix. Note that the Fock space dimension of the matrix is $2^{N}$, where $N$ is the total number of included single-electron states. In order to obtain a numerically practicable algorithm we will make some simplifying assumptions in the following considerations. A comparable Green's function approach incorporating all correlation effects, which are included in the densitymatrix approach, would require a huge amount of Green's functions of higher order and therefore lead to comparable numerical efforts.

\section{A. Thermodynamical state}

We are primarily interested in expectation values of observables concerning the quantum dot alone, especially the total electron number and the total spin. Thus we only need to know the projected density matrix

$$
\rho^{d o t} \equiv \operatorname{Tr}_{d o t}(\rho)
$$

of the quantum dot, where $\rho$ denotes the matrix of the whole reservoir-dot system. In the following we will assume two reservoirs $L$ and $R$ (for left and right). Then our approach reads

$$
\rho^{d o t} \simeq \alpha_{L} \rho_{L 0}^{d o t}+\alpha_{R} \rho_{R 0}^{d o t} \text { with } \alpha_{X}=\frac{\Gamma_{X}}{\Gamma_{L}+\Gamma_{R}},
$$

where $-\Gamma_{X} / 2$ is the imaginary part of the coupling selfenergy of reservoir $X$. Let us consider the case $\Gamma_{R} \ll \Gamma_{L}$. Then we have

$$
\rho^{d o t}=\rho_{L 0}^{d o t}+\delta \rho_{R}^{d o t}
$$

with the equilibrium form $\rho_{L 0}^{d o t}$. We assume that the small pertubation $\delta \rho_{R}^{d o t}$ gives rise only to second-order corrections in expectation values of the considered observable class.

\section{B. Interaction}

We will first discuss all the interaction terms arising from Coulomb repulsion and attraction of dot electrons, reservoir electrons, and the positively charged background (the socalled jellium) inside the reservoirs. Since the intradot electron-electron interaction plays a crucial role concerning many-particle exchange and correlations effects we will account for these terms in a fully quantum-mechanical treatment in second quantization. The remaining terms will be described in a semiclassical picture with a constant Hartree interaction energy. See Table I for a summary. $v$ denotes reservoir-reservoir terms and $\tilde{v}$ reservoir-dot terms. Here we consider expressions of the form
TABLE I. Interaction terms for a coupled reservoir-dot system. $v$ and $\tilde{v}$ denote capacitive Hartree matrix elements, $Q$ denotes the many-particle Coulomb term, $N$ the total electron number of the system, $n$ the intradot electron number.

\begin{tabular}{lcc}
\hline \hline Charge & Approach & Model term \\
\hline Jellium Jellium & Hartree & $\frac{N(N-1)}{2}$ \\
Reservoir $\leftrightarrow$ Reservoir & Hartree & $v \frac{(N-n)(N-n-1)}{2}$ \\
Jellium Reservoir & Hartree & $-v N(N-n)$ \\
Dot $\leftrightarrow$ Dot & Full & $Q(n)$ \\
Dot $\leftrightarrow$ Jellium & Hartree & $-\tilde{v} n N$ \\
Dot $\leftrightarrow$ Reservoir & Hartree & $\tilde{v} n(N-n)$ \\
\hline \hline
\end{tabular}

$$
v=\frac{1}{4 \pi \epsilon_{0} \epsilon_{r}} \int d^{3} x \int d^{3} x^{\prime} \frac{n_{A}(\vec{x}) n_{B}\left(\vec{x}^{\prime}\right)}{\left|\vec{x}-\vec{x}^{\prime}\right|}
$$

for the Hartree term between semiclassical charge distributions $n_{A}$ and $n_{B}$ (normalized to $1 e$ ) in two regions $A$ and $B$, respectively. Note that we make a Hartree approximation only for those interacting subsystems where reservoir charges are involved. Charges inside the reservoirs are thermalized and therefore correlation and exchange terms can be neglected, so that a description within the scope of the Hartree approximation is adequate.

Summarizing these terms we can write for the total interaction energy $E_{I}$ of a quantum dot and one reservoir in our model

$$
E_{I}=\underbrace{\frac{1}{2} v}_{=\gamma_{1}} n+\underbrace{\left(\frac{1}{2} v-\tilde{v}\right)}_{=\gamma_{2}} n^{2}+\underbrace{Q(n)}_{\rightarrow C}
$$

In the following section we will refer to the prefactors as $\gamma_{1}$ and $\gamma_{2}$. The quantization of the first semiclassical expression consists in the introduction of particle number operators, whereas the last many-particle term will be determined by a Coulomb tensor $C$ expression.

\section{Model Hamiltonian}

The total model Hamiltonian for the quantum dot thus reads

$$
H=\sum_{i, j} \epsilon_{i j} b_{i}^{\dagger} b_{j}+\gamma_{1} \hat{N}+\gamma_{2} \hat{N}^{2}+\frac{1}{2} \sum_{i, j, k, l} C_{i j k l} b_{i}^{\dagger} b_{j}^{\dagger} b_{k} b_{l}
$$

with single-particle matrix elements $\epsilon_{i j}$ and with the Coulomb tensor

$$
\begin{aligned}
C_{i j k l}= & \frac{e^{2}}{4 \pi \epsilon_{0} \epsilon_{r}} \sum_{\sigma, \sigma^{\prime}} \int d^{3} x \int d^{3} x^{\prime} \phi_{i}^{*}(\vec{x}, \sigma) \phi_{j}^{*}\left(\vec{x}^{\prime}, \sigma^{\prime}\right) \\
& \times \frac{1}{\left|\vec{x}-\vec{x}^{\prime}\right|} \phi_{k}\left(\vec{x}^{\prime}, \sigma^{\prime}\right) \phi_{l}(\vec{x}, \sigma) .
\end{aligned}
$$


Here $b_{i}$ and $b_{i}^{\dagger}$ denote annihilation operators referring to an arbitrary but numerically suitable set of single-particle states $\left\{\phi_{i}\right\}$. The index $i$ includes spin and spatial degrees of freedom. $\hat{N}$ is the particle number operator. Note that

$$
C_{i j k l} \propto \delta_{\mathrm{spin}(i) \operatorname{spin}(l)} \delta_{\operatorname{spin}(j) \operatorname{spin}(k)} .
$$

For an arbitrarily given attractive potential $V$ and an external magnetic field (in the $z$ direction) with cyclotron frequency $\omega_{c}$ the Rashba ${ }^{15}$ single-particle matrix elements in a semiconductor heterostructure are taken to be

$$
\begin{aligned}
\epsilon_{i j}= & \left\langle\phi_{i},\left[-\frac{\hbar^{2}}{2 m^{*}} \Delta+V(x, y)\right.\right. \\
& -i \hbar \omega_{c} x \frac{d}{d y}+\frac{1}{2} m^{*} \omega_{c}^{2} x^{2} \\
& \left.\left.+\frac{1}{\hbar} \vec{\alpha} \cdot(\vec{S} \times \vec{\pi})+\frac{g^{*} m^{*} \hbar \omega_{c}}{2 m_{e}} S_{z}\right] \quad \phi_{j}\right\rangle .
\end{aligned}
$$

$\vec{S}$ denotes the electron spin operator, $\vec{\alpha}$ the Rashba coupling coefficient, $\vec{\pi}$ the kinetic momentum, and $m^{*}$ the electron effective mass.

For numerical reasons we restrict ourselves to a finite subset $B:=\left\{\phi_{i} \mid E_{i}<E_{\max }\right\}$ of the energetically lowest-lying single-particle states. First of all we diagonalize the singleparticle matrix $\epsilon_{i j}$ and obtain the orthonormalized eigenvector set $B^{\prime}:=\left\{\phi_{i}^{\prime}\right\}$ by use of a unitary transformation $P\left(P^{-1}=P^{\dagger}\right)$ :

$$
\phi_{i}^{\prime}=\sum_{j} P_{j i} \phi_{j}, \quad b_{j}=\sum_{i} P_{j i} b_{i}^{\prime} .
$$

Then we have $\epsilon=P \epsilon^{\prime} P^{-1}$ with the diagonal matrix $\epsilon_{p q}^{\prime}$ $=\delta_{p q} \epsilon_{p}^{\prime}$. The Coulomb tensor transforms as

$$
C_{p q r t}^{\prime}=\sum_{i, j, k, l} P_{i p}^{*} P_{j q}^{*} P_{k r} P_{l t} C_{i j k l}
$$

In order to keep the Fock space dimension $2^{N}$ as small as possible for numerical reasons we truncate $B^{\prime}$ to a new restricted base set $B^{\prime \prime}=\left\{\phi_{i}^{\prime} \in B^{\prime} \mid \epsilon_{i}^{\prime}<\widetilde{E}_{\max }<E_{\max }\right\} \subseteq B^{\prime}$. Note that we have to ensure that the choice of $\widetilde{E}_{\text {max }}$ does not change the expectation values significantly for a given chemical potential. Here one must keep in mind that the interaction is repulsive and therefore energetically raises the electron filling of the quantum dot.

For this given single-particle base $B^{\prime \prime}$ we construct orthonormalized field states

$$
\Phi_{I}=\left(b_{N-1}^{\prime \dagger}\right)^{n_{N-1}} \cdots\left(b_{1}^{\prime \dagger}\right)^{n_{1}}\left(b_{0}^{\prime \dagger}\right)^{n_{0}} \Phi_{v a c}
$$

starting from the vacuum state $\Phi_{v a c}$. Then we have to evaluate Fock space matrix elements $M_{J I}$ of the form

$$
M_{J I}=\left(\Phi_{J}, b_{x}^{\prime \dagger} b_{x}^{\prime} \Phi_{I}\right)
$$

and further

$$
\widetilde{M}_{J I}=\left(\Phi_{J}, b_{x}^{\prime \dagger} b_{y}^{\prime \dagger} b_{z}^{\prime} b_{w}^{\prime} \Phi_{I}\right)
$$

Note that anticommutation symmetries drastically reduce the number of independent elements.

Now we can write down the many-particle density matrix $\rho^{d o t}$ for the temperature $T$ (with $\beta:=1 / k_{B} T$ ) and the chemical potential $\mu$ :

$$
\rho^{d o t}=\frac{1}{Z_{g r}} \exp [-\beta(H-\mu \hat{N})],
$$

with the particle number operator $\hat{N}=\sum_{i} b_{i}^{\prime \dagger} b_{i}^{\prime}$ and the grand-canonical partition function

$$
Z_{g r}=\operatorname{Tr}\{\exp [-\beta(H-\mu \hat{N})]\}
$$

In the following we will use the abbreviation $\hat{H}:=-\beta(H$ $-\mu \hat{N})$. In order to calculate the exponential function we diagonalize $\hat{H}$ with a unitary (Fock space) transformation $Q$ :

$$
\hat{H}_{\text {diag }}=Q^{-1} \hat{H}_{B^{\prime \prime}} Q
$$

Then we have

$$
\begin{gathered}
\exp \left(\hat{H}_{B^{\prime \prime}}\right)=Q \exp \left(\hat{H}_{\text {diag }}\right) Q^{-1} \\
\left(\exp \left(\hat{H}_{\text {diag }}\right)\right)_{I J}=\delta_{I J} \exp \left[\left(\hat{H}_{\text {diag }}\right)_{I I}\right]
\end{gathered}
$$

Note that $\exp \left[\left(\hat{H}_{\text {diag }}\right)_{I I}\right]$ is a real number. Finally we obtain

$$
Z_{g r}=\sum_{I} \exp \left[\left(\hat{H}_{\text {diag }}\right)_{I I}\right]
$$

and

$$
\rho_{B^{\prime \prime}}^{d o t}=\frac{1}{Z_{g r}} Q \exp \left(\hat{H}_{d i a g}\right) Q^{-1} .
$$

\section{Resonant tunneling diode}

A resonant tunneling diode ${ }^{16}$ consists of a double barrier semiconductor heterostructure, that is, a one-dimensional (1D) quantum well with electron reservoirs coupled to both sides via tunnel barriers. Let us denote this direction as the $z$ direction. If one applies an additional confinement by use of an attractive potential in the lateral $(x, y)$ directions a threedimensional (3D) binding potential arises. ${ }^{2,17}$ On a mesoscopic length scale such a system represents a quantum dot with two directly coupled reservoirs. In order to have one dominating reservoir we just have to choose a strong barrier thickness asymmetry in the heterostructure.

Since in most experimental RTD situations of a disklike shaped quantum dot the spatial extent of the wave functions between the two barriers is about one order of magnitude smaller than the lateral dimensions, we only consider the ground state $n_{z}=0$ referring to the $1 \mathrm{D}$ quantum well in the $z$ direction. In this case a suitable orthonormalized singleelectron base set $B$ consists of $2 \mathrm{D}$ harmonic oscillator states ${ }^{18}$ with the characteristic lengths $x_{0}=\sqrt{\hbar /\left(\omega_{x 0} m^{*}\right)}$ and $y_{0}=\sqrt{\hbar /\left(\omega_{y_{0}} m^{*}\right)}$ and Hermite polynomials $H_{n}$ : 


$$
\begin{aligned}
\phi_{n_{x}, n_{y}, n_{z}=0, s}(x, y, z, \sigma) & \\
= & \delta_{\sigma s} \frac{1}{\sqrt{2^{\left(n_{x}+n_{y}\right)} n_{x} ! n_{y} ! \pi x_{0} y_{0}}} \\
& \times \exp \left\{-\frac{1}{2}\left[\left(\frac{x}{x_{0}}\right)^{2}+\left(\frac{y}{y_{0}}\right)^{2}\right]\right\} \\
& \times H_{n_{x}}\left(\frac{x}{x_{0}}\right) H_{n_{y}}\left(\frac{y}{y_{0}}\right) \sqrt{\frac{2}{z_{0}}} \sin \left(\frac{\pi z}{z_{0}}\right) .
\end{aligned}
$$

Note that these are not eigenfunctions of the single-particle part of the Hamiltonian in general. In the next section we will employ these base functions for numerical simulations.

\section{NUMERICAL RESULTS}

Based on the quantum dot model and the density-matrix description of the previous section we now discuss some basic aspects of electron filling characteristics inside RTD quantum dots. In order to study charging effects we assume a diode with strong barrier asymmetry under bias conditions where electrons accumulate inside the dot (that is, the emitter barrier must be thinner). As can be seen from Green's function calculations, the successive filling of the quantum dot with varying bias voltage (i.e. varying chemical potential) can be observed directly in the form of single-electron current steps. ${ }^{9}$ Thus there is a simple experimental means to measure electron occupation inside the quantum dot under the strong accumulation condition as a function of the chemical potential, which can be deduced directly from the applied bias voltage between the two reservoirs for a given magnetic field. For the influence of the external magnetic field on the chemical potential inside a reservoir, see Ref. 17.

In order to visualize the main aspects of the electronelectron interaction we first consider a GaAs RTD with a quantum well width of $z_{0}=17 \mathrm{~nm}\left(\mathrm{Al}_{0.3} \mathrm{Ga}_{0.7} \mathrm{As}\right.$ barriers $)$ and a 2D lateral harmonic potential with $\hbar \omega_{x 0}=\hbar \omega_{y_{0}}$. Further we assume $m^{*}=0.067 m_{e}, g^{*}=0.44, \epsilon_{r}=13.1$, and a temperature of $T=30 \mathrm{mK}$. For the ground state and the first excited state of the quantum well in the $z$ direction one easily obtains $E_{z 0}=20 \mathrm{meV}$ and $E_{z 1}=72 \mathrm{meV}$, respectively. Therefore we can consider a chemical potential range of $\Delta \mu=52 \mathrm{meV}$ if we restrict ourselves to a single-particle base set with the $z$-well ground state in the following. In the numerical part we take 18 single-particle base states (in $B$ ) and a 512-dimensional Fock subspace. A comparison between 1024 and 2048 dimensions shows that the deviations in the electron numbers are less than $5 \%$. The Coulomb matrix elements have been computed using Monte Carlo techniques, all single-particle matrix elements using standard numerical integration techniques. We will refer to the groundstate Coulomb interaction energy as the "Coulomb matrix element' $C_{0000}$ in the following.

Constant capacitive energy terms arising from the reservoirs and spin-orbit interaction terms will be set to zero in our examples since the major effects of many-particle correlation we are interested in arise from the intradot interaction. (The Rashba terms only provide minor corrections to the filling step positions concerning the chemical potential for the considered $\mathrm{Al}_{x} \mathrm{Ga}_{1-x} \mathrm{As} / \mathrm{GaAs}$ heterostructure. On the
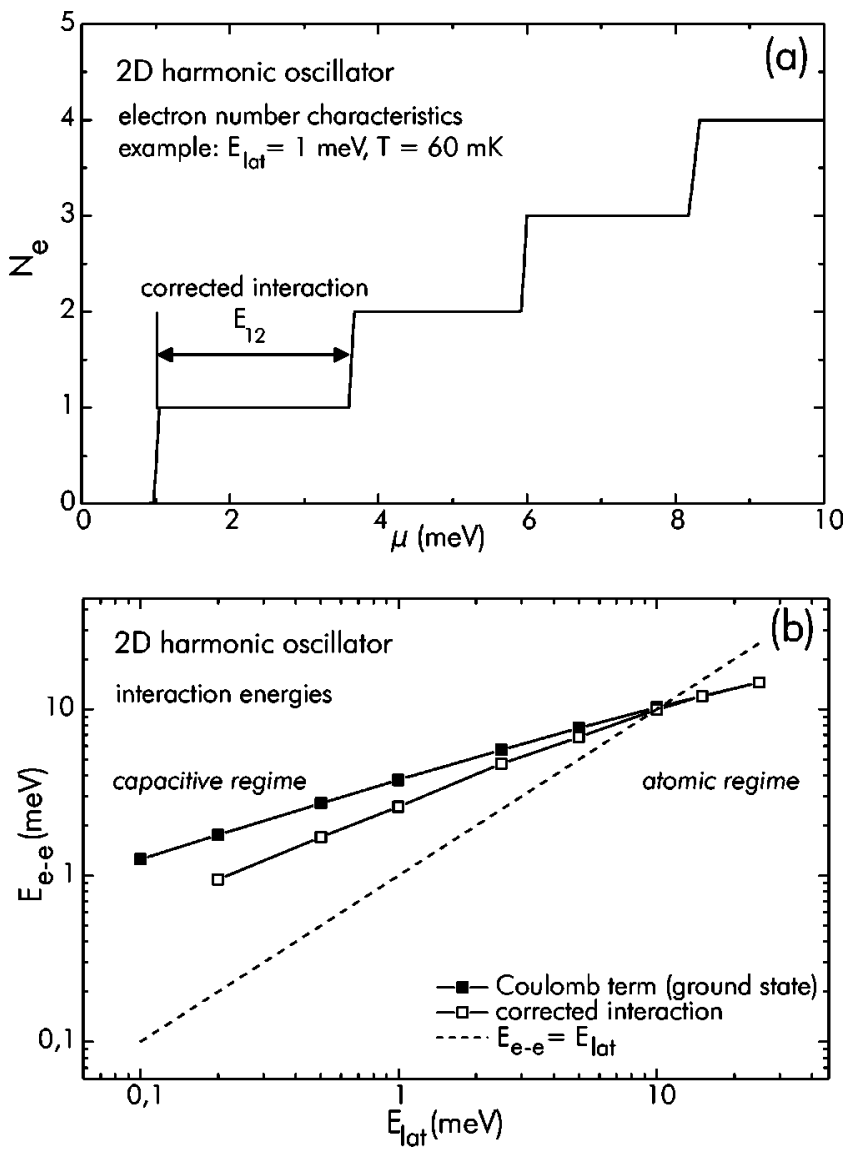

FIG. 1. Simulation overview. (a) Particle number $N_{e}$ versus chemical potential $\mu$ characteristics with a definition for the corrected interaction energy $E_{12}$. (b) Interaction energies $E_{e-e}$ as a function of the lateral quantization energy $E_{\text {lat }}$. Solid squares indicate the Coulomb term $C_{0000}$ of the single-particle ground state and open squares the corrected interaction energy $E_{12}$.

other hand, capacitive reservoir interaction terms can be estimated to be at least one order of magnitude smaller than the intradot terms for the RTD under consideration).

Figure 1(a) shows a simulated particle number versus chemical potential characteristics, where $E_{12}$ is defined as the "corrected interaction energy" of the first two electrons. In Fig. 1(b) various interaction energies are plotted for varying lateral quantization energy $E_{\text {lat }} \equiv \hbar \omega_{0}$.

First we consider the behavior of $C_{0000}$. We have $E_{\text {lat }}$ $=\hbar^{2} /\left(m^{*} x_{0}^{2}\right) \propto x_{0}^{-2}$, where $x_{0}$ denotes the lateral characteristic length, and further $C_{0000} \propto x_{0}^{-1}$ for large $x_{0}$ (that is, small $\left.E_{\text {lat }}\right)$. Therefore $C_{0000} \propto E_{\text {lat }}^{1 / 2}$, which can be seen in Fig. 1.

For $E_{l a t}>C_{0000}$ the Coulomb repulsion is larger than the single-particle level spacing and thus it is energetically favorable for the first two electrons to occupy the ground state with opposite spins. Hence we see that the corrected interaction energy $E_{12} \approx C_{0000}$ for this case. We will call this regime the "atomic regime."

On the other hand, for $E_{\text {lat }} \ll C_{0000}$ it is favorable to occupy a mixture of higher excited single-particle states because their interaction tensor elements diminish for growing quantum numbers. Therefore we observe $E_{12}<C_{0000}$ due to many-particle effects (Fig. 1). We will call this regime the "capacitive regime" since we observe a behavior analogous to a classical capacitor: Electrons tend to distribute their total 

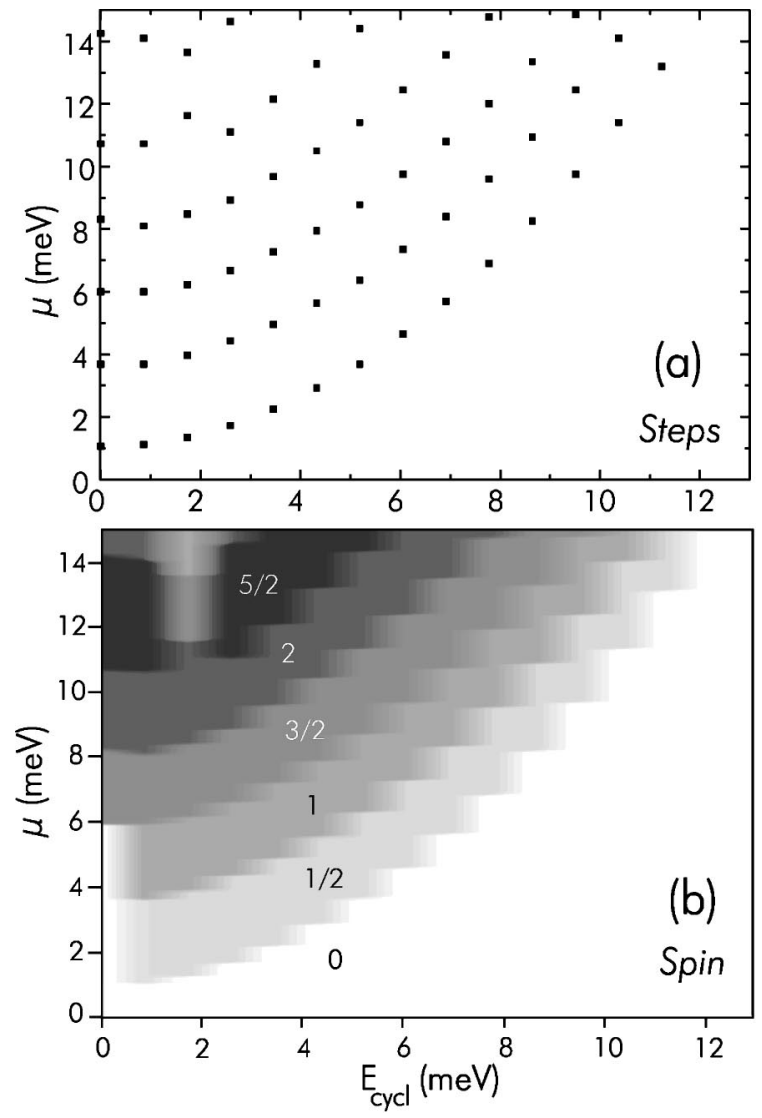

FIG. 2. Capacitive regime. (a) Simulated positions of singleelectron steps at chemical potenial $\mu$ as a function of an external magnetic field with cyclotron energy $E_{c y c l}$. (b) Total electron spin in $z$ direction represented as a gray-scale plot. (Steplike borders are due to the simulation step width in $E_{c y c l}$. A bright structure in the region of spin $5 / 2$ is an artifact due to the finite Fock subspace dimension.)

charge on the surface of the confining potential, which can readily be seen from the extent $\Delta x_{j} \propto \sqrt{2 j+1}$ of an excitedstate wave function for quantum number $j$. In the next two subsections we will discuss some electron filling details of each quantum dot regime (analogous to Hund's rules for atoms).

\section{A. Capacitive regime}

This regime requires a large amount of computational power since with diminishing $E_{l a t}$ we have to include a lot of single-particle levels within the interaction energy $C_{x y y x}$ to add one additional electron to the quantum dot system. Therefore the dimension of the Fock subspace grows exponentially. Now we will consider the above mentioned RTD quantum dot with $E_{\text {lat }}=1 \mathrm{meV}\left(\Rightarrow C_{0000} \approx 3.8 \mathrm{meV}, E_{12}\right.$ $\approx 2.6 \mathrm{meV}$ ). Hereby we have taken 50 single-particle levels as a base set and a Fock space dimension of 512 up to 1024 .

In Fig. 2(a) one can see the position of single-electron steps as a function of an externally applied magnetic field in the $z$ direction with cyclotron energy $E_{c y c l} \equiv \hbar \omega_{c}$ $\equiv \hbar e B / m^{*}$. For the first step at the lowest chemical potential we observe the typical magnetic field dependence of the ground state of a 2D harmonic oscillator. ${ }^{19}$ Furthermore we observe a nearly parallel shift of all following steps with
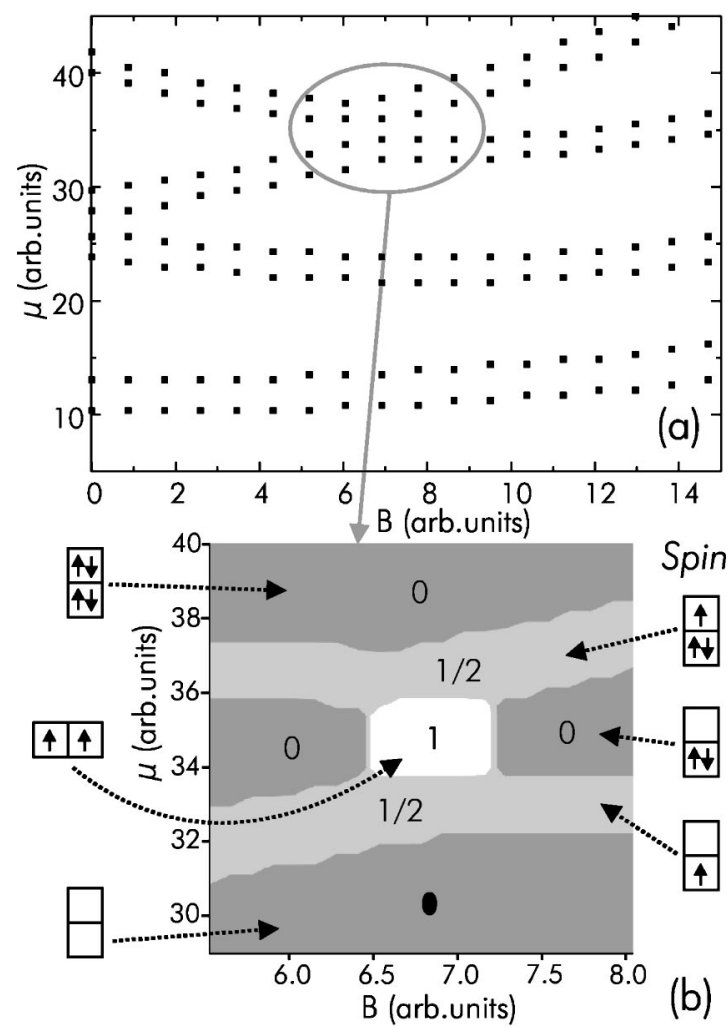

FIG. 3. Atomic regime. (a) Simulated positions of singleelectron steps at chemical potential $\mu$ as a function of an external magnetic field $B$. (b) Total electron spin in the $z$ direction represented as a gray-scale plot in the vicinity of a degeneracy point of single-particle levels.

varying magnetic field. The difference in chemical potential for adjacent steps is quite similar over the full field range. This behavior is typical for the capacitive regime where the repulsion energy is larger than the single-particle level spacing. Thus even in this system of a few strong interacting and correlated electrons one obtains a semiclassical filling behavior equivalent to a capacitor. The orthodox theory ${ }^{10}$ (with the above-defined $E_{12}$ as the effective interaction energy) therefore can be applied to some degree for the description concerning the total particle number. Hence we are able to define an intradot interaction capacity $C_{12}=e^{2} / E_{12}$ for use with the orthodox theory based on $E_{12}$.

Obviously the density-matrix approach provides complete information about the quantum-mechanical state and the discussed class of observables. An interesting feature of the capacitive regime can be seen in Fig. 2(b) where the total electron spin in the $z$ direction is plotted in a gray-scale diagram. Due to exchange-correlation effects we observe a polarization of the electron system in the applied magnetic field.

Experimental evidence for such a capacitive behavior of single-electron steps in an external magnetic field can be found in various publications. ${ }^{20}$ Here laterally confined RTD structures have been investigated for the case of strong barrier asymmetry similar to the numerically simulated quantum dot in this example. By use of a Schottky gate for a variable lateral confinement the electronic filling of a RTD quantum dot has be investigated ${ }^{21}$ as a function of the lateral potential (with fixed chemical potential $\mu$ ). For an arbitrarily given 
lateral potential $V$ (i.e., single-particle matrix elements as a function of the gate voltage) in the Hamiltonian equation (2.6) a numerical treatment of such systems should be possible within the scope of the density matrix approach even in the case of strong intradot correlations due to many-particle interaction effects.

\section{B. Atomic regime}

In contrast to the capacitive regime we now consider the case where the single-particle level spacing $E_{\text {lat }}$ is larger than the first Coulomb tensor element $C_{0000}$. This regime can be reached in semiconductor systems only in the case of quantum dots on a characteristic length scale smaller than a few tens of nanometers. Figure 3 shows a typical filling behavior of an artifical quantum dot with $E_{l a t}=10 \mathrm{meV}$ and $C_{0000} \approx 2 \mathrm{meV}$. Again the first lowest-lying step exhibits the characteristic $B$-field dependence followed by the second spin-degenerate electron at $\Delta \mu_{12} \equiv C_{0000}$. Higher singleparticle levels ${ }^{19}$ still can be recognized with an interactioninduced splitting of the spin degeneracy. As an interesting feature we now focus on the four-level anticrossing point that is visualized in Fig. 3(b). Hereby the total spin is shown as a gray-scale plot. Only in the center of the anticrossing does one observe a parallel spin alignment due to small energy lowering electron exchange terms. At all other points the system shows a tendency to minimize the total spin in the $z$ direction for small magnetic fields. Some primitive orbital figures show an atomlike behavior.

\section{CONCLUSION}

Based on a many-particle density-matrix approach with an underlying interaction Hamiltonian in second quantization we have been able to describe the electron filling characteristics of an interacting quantum dot system. Especially intradot exchange and correlation effects have been incorporated. Employing numerical results for the case of a single-electron RTD in the strong accumulation regime we define a capacitive and atomic quantum dot depending on the ratio between size quantization energies and interaction matrix elements, that is, the geometrical dimensions. We presented a fully quantum-mechanically based definition of an intradot interaction capacity. A subsequent paper is planned to present experimental results on a RTD quantum dot system and a comparative analysis based on the density-matrix model.

${ }^{1}$ Single Charge Tunneling, edited by H. Grabert and M. Devoret, NATO Advanced Study Institute, Series B: Physics (Plenum, New York, 1992).

${ }^{2}$ M. A. Reed, J. N. Randall, R. J. Aggarwal, R. J. Matyi, T. M. Moore, and A. E. Wetsel, Phys. Rev. Lett. 60, 535 (1988); A. Groshev, Phys. Rev. B 42, 5895 (1990); J. N. Randall, M. A. Reed, T. M. Moore, R. J. Matyi, and J. W. Lee, J. Vac. Sci. Technol. B 6, 302 (1988); M. Luban, J. H. Luscombe, M. A. Reed, and D. L. Pursey, Appl. Phys. Lett. 54, 1997 (1989).

${ }^{3}$ W. R. Frensley, Phys. Rev. B 36, 1570 (1987).

${ }^{4}$ C. Caroli, R. Combescot, P. Nozieres, and D. Saint-James, J. Phys. C 4, 916 (1971).

${ }^{5}$ R. Lake, G. Klimeck, R. C. Bowen, and D. Jovanovic, J. Appl. Phys. 81, 7845 (1997).

${ }^{6}$ G. D. Mahan, Many-Particle Physics, 2nd ed. (Plenum, New York, 1990); E. N. Economou, Green's Functions in Quantum Physics, 2nd ed. (Springer Verlag, Heidelberg, 1983).

${ }^{7}$ L. E. Henrickson, A. J. Glick, G. W. Bryant, and D. F. Barbe, Phys. Rev. B 50, 4482 (1994).

${ }^{8}$ A. Groshev, T. Ivanov, and V. Valtchinov, Phys. Rev. Lett. 66, 1082 (1991); Y. Meir, N. S. Wingreen, and P. A. Lee, ibid. 66, 3048 (1991); L. Y. Chen and C. S. Ting, Phys. Rev. B 44, 5916 (1991).

${ }^{9}$ K. M. Indlekofer, J. Lange, A. Förster, and H. Lüth, Phys. Rev. B 53, 7392 (1996).

${ }^{10}$ C. W. Beenakker, Phys. Rev. B 44, 1646 (1991).

${ }^{11}$ J. J. Palacios, L. Martín-Moreno, J. H. Oaknin, and C. Tejedor, Superlattices Microstruct. 15, 91 (1994).

${ }^{12}$ D. Pfannkuche, V. Gudmundson, and P. A. Maksym, Phys. Rev. B 47, 2244 (1993).

${ }^{13}$ U. Fano, Rev. Mod. Phys. 29, 74 (1957).

${ }^{14}$ A. Barenco and M. A. Dupertuis, Phys. Rev. B 52, 2766 (1995).

${ }^{15}$ Y. A. Bychkov and E. I. Rashba, Pis'ma Zh. Eksp. Teor. Fiz. 39, 66 (1984); E. I. Rashba, Fiz. Tverd. Tela (Leningrad) 2, 1224 (1960).

${ }^{16}$ R. Tsu and L. Esaki, Appl. Phys. Lett. 22, 562 (1973); L. L. Chang, L. Esaki, and R. Tsu, ibid. 24, 593 (1974).

${ }^{17}$ M. Griebel, K. M. Indlekofer, A. Förster, and H. Lüth, J. Appl. Phys. 84, 6718 (1998); A. Förster, M. Griebel, M. Indlekofer, and H. Lüth, Physica E (Amsterdam) 2, 502 (1998).

${ }^{18}$ D. Pfannkuche and S. E. Ulloa, Phys. Rev. Lett. 74, 1194 (1995).

${ }^{19}$ V. Fock, Z. Phys. 47, 446 (1928).

${ }^{20}$ M. Tewordt, V. J. Law, J. T. Nicholls, L. Martín-Moreno, D. A. Ritchie, M. J. Kelly, M. Pepper, J. E. F. Frost, R. Newbury, and G. A. C. Jones, Solid-State Electron. 37, 793 (1994); T. Schmidt, M. Tewordt, R. H. Blick, R. J. Haug, B. Pfannkuche, K. v. Klitzing, A. Förster, and H. Lüth, Phys. Rev. B 51, 5570 (1995); B. Su, V. J. Goldman, and J. E. Cunningham, Surf. Sci. 305, 566 (1994).

${ }^{21}$ S. Tarucha, D. G. Austing, T. Honda, R. v. d. Hage, and L. P. Kouwenhoven, Jpn. J. Appl. Phys., Part 1 36, 3917 (1997); M. W. Dellow, P. H. Beton, C. J. G. M. Langerak, T. J. Foster, P. C. Main, L. Eaves, M. Henini, S. P. Beaumont, and C. D. W. Wilkinson, Phys. Rev. Lett. 68, 1754 (1992); P. Guéret, N. Blanc, R. Germann, and H. Rothuizen, ibid. 68, 1896 (1992). 